\title{
Self-care behaviors and practices of nursing students: Review of literature
}

\author{
Ahtisham Younas ${ }^{1,2 *}$ \\ ${ }^{1}$ Memorial University of Newfoundland, Canada, ${ }^{2}$ Shifa College of Nursing, Islamabad, Pakistan
}

\begin{abstract}
Self-care is a vital component of nursing practice and is essential for the well-being of human beings, that is, both patients and nurses. However, nurses and nursing students tend to focus more on self-care of their patients and often pay little or no attention to personal self-care. This literature review explored selfcare behaviors and practices of nursing students in general as well as across academic years of the study. In total, 11 studies were reviewed and critically appraised to identify current trends regarding nursing students' self-care practices and behaviors in nursing literature. Nursing students realized the importance of maintaining their diet, sleep, and activity level to improve physical and physiological health. However, they tend to neglect self-care practices which could improve their emotional and psychological health because of academic stress, workload, and inadequate knowledge about the strategies to improve self-care in this domain. There is limited evidence for drawing any conclusions regarding students' use of complementary therapies for self-care, the difference between self-care practices of nursing and non-nursing students, the usefulness of different interventions for improving students' self-care practices, and the differences between self-care practices and behaviors of students across academic years.
\end{abstract}

Key words: student's self-care; nursing; behavior

\section{INTRODUCTION}

Self-care is an important constituent of nursing practice (1), and its significance in nursing was first made explicit by Dorothea Orem in her self-care theory. Orem explicated that self-care is essential for the well-being of human beings, that is, both patients and nurses (2). However, nurses and nursing students tend to focus more on nursing care of

\footnotetext{
*Corresponding author: Ahtisham Younas, Memorial University of Newfoundland, Canada. Shifa College of Nursing, Pitras Bukhari Road, Sector H-8/4, Islamabad-44000, Pakistan.

E-mail: ay6133@mun.ca
}

Submitted: 5 April 2017/Accepted: 30 August 2017

DOI: https://doi.org/10.17532/jhsci.2018.420 their patients and often pay little or no attention to personal self-care $(1,3)$. Without practicing, personal self-care nurses and nursing students cannot provide quality care to their patients (4).

The findings from the recent systematic and integrative reviews indicated that nursing students experience numerous stressors throughout their academic life $(5,6)$, which affect their abilities to care for themselves and their patients (7). It has also been found that nurses and nursing students who engage in selfcare are perceived positively by patients and their families. Furthermore, patients get encouragement from such nurses and nursing students and engage in self-care (8). Although both nurses and nursing students neglect self-care, nursing students being 
young and immature may lack the much-needed experience for self-care and health promotion practices (9). The purpose of this literature review was to explore self-care behaviors and practices of nursing students in general as well as across academic years of the study. This review will also determine the extent of research on self-care of nursing students and explore areas for future research.

\section{Literature search}

An electronic search was conducted within the databases: CINAHL, PubMed, Google Scholar, and Science Direct spanning the years 2000-2016 using the phrases "self-care behaviors of nursing students," "health-care practices of nursing students," and "selfcare practices of nursing students" and the keywords "care for self," "personal self-care," and "nursing students." This search retrieved 65 studies, pertinent to the specific terms, which were analyzed by reading titles, abstracts, and conclusions. Based on this screening and purposive sampling, 15 selected studies were read in depth to check for eligibility. Finally, 11 studies were selected for this review. The selection criteria were: (i) Original research studies (both qualitative and quantitative) in the English language and (ii) studies focusing exclusively on nursing students or nursing students in comparison with other students as the target population.

\section{Overview and critical appraisal of the studies}

All of the included studies clearly outlined their research question, purpose, target population, sample, and its characteristics. Out of 11 studies, five studies used cross-sectional design (1,9-12), two studies used longitudinal design $(13,14)$, two studies used experimental designs $(8,15)$, and one study each used qualitative (16) and action research method (17). Out of 11 studies, five studies guided the research through the lens of theoretical framework $(9,11,12,14,17)$. The sample size ranged from 15 to 300 and was recruited using convenient sampling. None of the studies used a random sample. Ethical approval and informed consent were obtained in all of the studies, and essential measures were taken to ensure confidentiality, privacy, and respect of the participants. All of the studies used valid and reliable data collection instruments except some researchers $(1,10,11,14)$. The researchers used appropriate methods for descriptive and inferential analyses. The detailed findings and strengths and limitations of the studies are presented in Table 1.

\section{FINDINGS}

The findings of this review were reported under seven categories, namely, physical and physiological selfcare behaviors and practices, substance abuse and driving, health screening practices, emotional and psychological health-care behaviors and practices, factors and interventions influencing health-care behaviors and practices, comparison of health-care practices of nursing and non-nursing students, and comparison of health behaviors and practices across academic years.

\section{Physical and physiological behaviors and practices}

The physical and physiological behaviors and practices of nursing students was the most repetitive theme in most of the studies (1,9-17). Based on the findings of this review, it was defined as the behaviors or practices concerning diet or nutrition, exercise or physical activities, and sleeping habits of students. All of the studies under this theme reported healthy self-care behaviors of students except two studies $(1,10)$. Among the studies that reported the positive findings, Horneffer (11) found that, out of 300 students, $58 \%$ of students exercised regularly while only $4 \%$ did not exercise. Nevins and Sherman (2016) found that, out of 119 students, $77.7 \%$ ate a balanced diet while $22.6 \%$ rarely ate balanced diet, $62 \%$ of students reported drinking about 3-8 glasses of water daily, 34\% exercised regularly, and $24.5 \%$ exercised rarely, but $70 \%$ of students did not exercise enough. Consistently, Chow and Kalischuk (12) found that, out of 211 students, $83 \%$ used to sleep 6-8 hours at the night; $60 \%$ reported that the sleep was adequate, whereas $37 \%$ reported inadequate sleep, 65\% students reported drinking four to eight glasses of water or juice a day, $77 \%$ students ate balanced diet (49\% "frequently" and 28\% "consistently"), and $71 \%$ students exercised regularly or occasionally, whereas $4 \%$ did not exercise at all. Clément et al. (13) observed self-care practices of students for 3 consecutive years: 1992, 1993, and 1994. The authors reported that majority of the students reported having adequate sleep (1992 = 


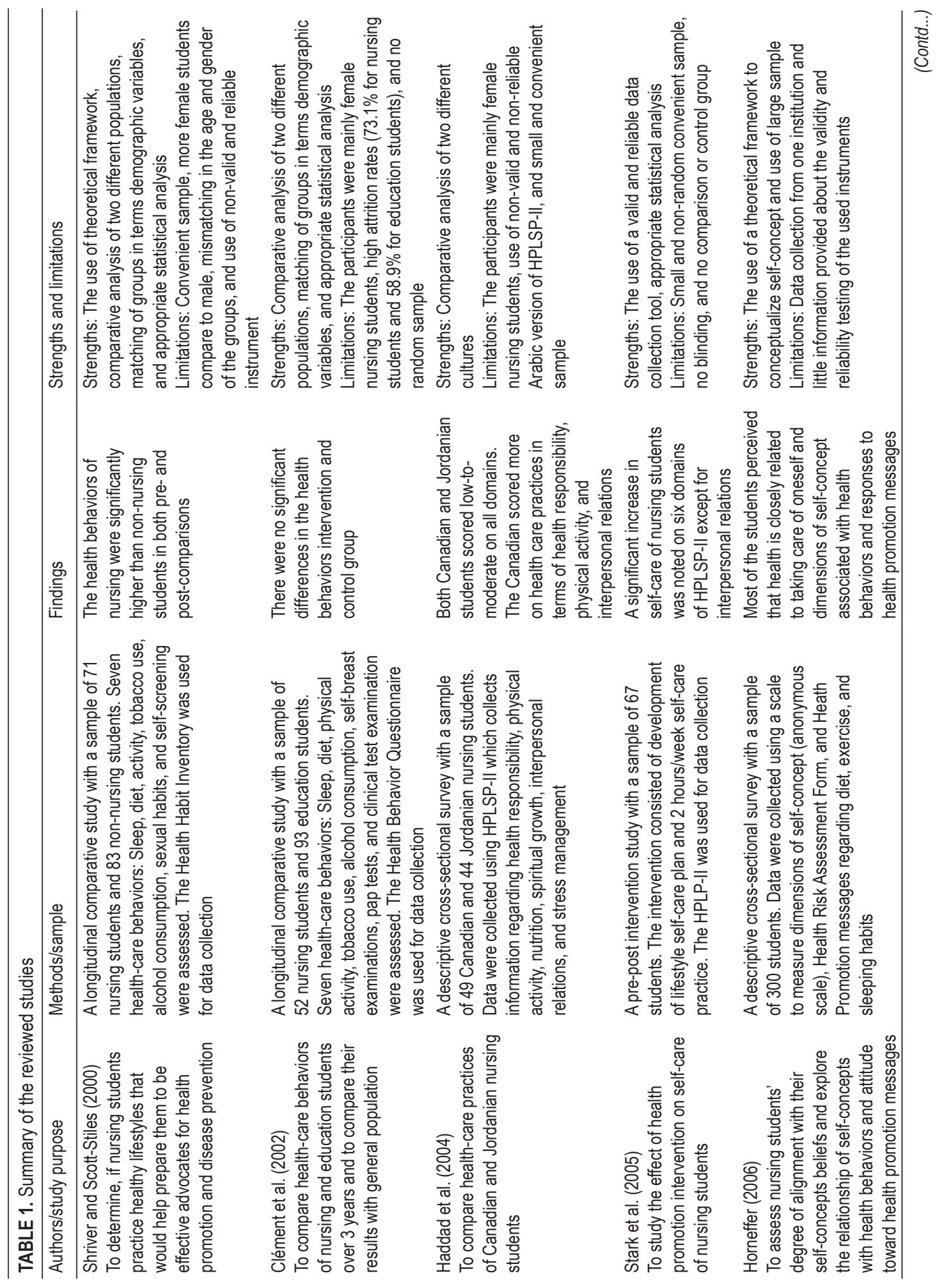




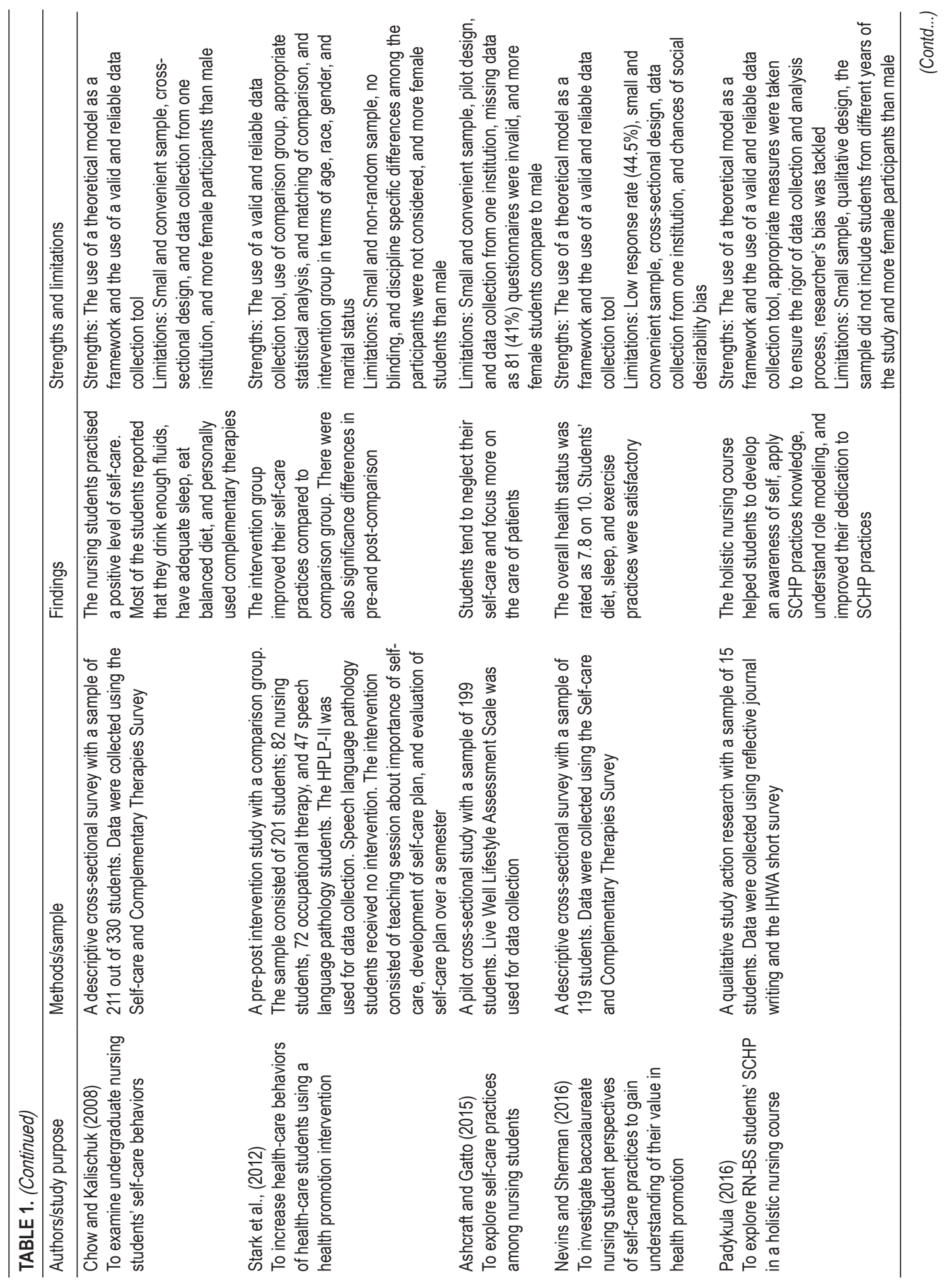


$73 \%, 1993=79 \%$, and $1994=71 \%$, eating balanced diet $(1992=88 \%, 1993=81 \%$, and 1994 $=79 \%)$, and carrying out adequate exercise (1992 $=81 \%, 1993=81 \%$, and $1994=67 \%)$. Similar findings were reported by other researchers $(8,14-$ 17). However, among the studies reporting the negative findings, Ashcraft and Gatto (1) and Haddad et al. (10) reported that students had low-to-moderate self-care behaviors. The mean self-care practices on health responsibility, physical activity, and nutrition ranged from 2.07 to 2.58 indicating low self-care practices (10). In general, the evidence suggests that students have good self-scare practices in terms of nutrition, sleep, drinking water, and physical activity. Siappo's et al. (16) qualitative findings affirm this because the students in their study realized the importance of balanced diet, active lifestyle, adequate sleep, and body hygiene in maintaining their self-care.

\section{Substance abuse and driving practices}

Several studies reported substance abuse including tobacco, alcohol, and illegal drugs and driving habits of nursing students (11-14,16). In general, all of the studies reported that nursing students avoided smoking, alcohol consumption, drug abuse, and used safety measures while driving. For example, Siappo's et al. (16) reported that students did not want to use tobacco and drugs because they considered them a threat to their health and security. Horneffer (11) reported that $71 \%$ of students never smoked and $18 \%$ never consumed alcohol. However, $5 \%$ of students who smoked were not interested in quitting and 38\% who consumed alcohol did not intend to refrain from it. Chow and Kalischuk (12) found that $59 \%$ of students consumed alcohol occasionally, whereas $35 \%$ did not consume at all, and $85 \%$ of students were non-smokers. This was the highest percentage of alcohol consumption in all the reviewed studies. On the other hand, Clément et al. (13) found that, over a period of 3 years, $80-93 \%$ of students did not consume alcohol, $80-90 \%$ abstained from smoking, and $94-90 \%$ wear seatbelts while driving. Shriver and Scott-Stiles (14) assessed self-care practices of 71 nursing students over 2 years. The researchers found that there was an improvement in the self-care behaviors of nursing students regarding alcohol and illegal drug use; in 
the first year, $9.9 \%$ of students consumed alcohol and $1.4 \%$ used illegal drugs, whereas in the second year, $8.8 \%$ consumed alcohol and $0 \%$ used illegal drugs. Regarding driving habits, an improvement was also seen; in the first year, $57.7 \%$ always wear seat belt as drivers and $39.4 \%$ as passengers, whereas in the second year, this percentage increased to $77.2 \%$ and $57.9 \%$, respectively. On the other hand, the students smoked more in the second year (8.8\%) compared to first year (7.0\%). However, the results of this study should be generalized with caution due to $9.94 \%$ attrition of nursing students in the second year.

\section{Health screening practices}

Health screening practices including Pap smear, selfbreast examination, self-testicular examination, and general screening were assessed by only two studies. Clément et al. (13) assessed self-care behaviors of students concerning self-breast examinations, clinical breast examination, and Pap smear. The researchers found that high percentage of nursing students engaged in clinical breast examination (1992 =75\%, $1993=79 \%$, and $1994=77 \%)$ and Pap smear $(1992=67 \%, 1993=69 \%$, and $1994=81 \%)$ compared to self-breast examination $(1992=27 \%, 1993$ $=41 \%$, and $1994=43 \%)$. Shriver and Scott-Stiles (14) found that the percentage of students engaged in most of health screening practices increased from first year to second year. For example, self-breast examination (23.3-33.3\%), self-testicular examination (0-33.3\%), and blood pressure monitoring (83.1-87.7\%). However, there was a slight decrease in some areas such as cholesterol monitoring (31.0$29.8 \%$ ) and safe sex practices (63.4-50.9\%). In general, the results are mixed, but the findings indicate that students engage in health screening and realize its importance in maintaining self-care.

\section{Emotional and psychological behaviors and practices}

Several studies discussed the emotional and psychological self-care behaviors and practices of students $(1,8-12,17)$. For this review, such practices entailed stress management, spiritual growth, interpersonal relations, and the use of complementary therapies. Haddad et al. (10) reported low scores on spiritual growth, interpersonal relations, and stress management of both Canadian and Jordanian nursing students with mean scores: Spiritual growth (2.97 vs. 2.98$)$, interpersonal relations (3.12 vs. 2.78 ), and stress management (2.46 vs. 2.58). These scores indicated that students did not engage in healthy emotional and psychological self-care practices. Contrary to these findings, Stark et al. (15) reported generally good mean scores on two domains such as spiritual growth (3.27) and interpersonal relations (3.43), and low scores on stress management (2.53). Regarding emotional self-care, Padykula (17) assessed emotional well-being of students and reported a high mean score of 4.12.

With regard to the use of complementary therapies by students, Nevins and Sherman (9) found that, out of 119 students, $45 \%$ actively used complementary therapies such as yoga, music, and meditation, whereas $54 \%$ denied using such therapies. This high percentage of students not using complementary therapies could be due to their lack of knowledge. The researchers reported that students rated their knowledge about such therapies 5.5 on the scale of 10 . Chow and Kalischuk (12) also found that, out of 211 students, $76 \%$ of students used complementary therapies for their emotional and psychological well-being. The students mainly used complementary therapies: Massage (54\%), vitamins (49\%), chiropractic (25\%), herbal medicine $(24 \%)$, yoga $(21 \%)$, aromatherapy (18\%), and acupuncture (9\%). Based on mixed findings under this theme, it could be implied that the data are insufficient to reach a conclusion as to what extent students engage in self-care practices that promote their emotional and psychological well-being.

\section{Factors/interventions influencing self-care behaviors and practices}

Several factors and interventions were reported to influence self-care practices and behaviors of students $(1,8-13,15-17)$. The common factors were cultural beliefs, perceptions about health, watching awareness programs about self-care on TV (10), academic and clinical stress and workload $(13,16)$, and increased knowledge of diseases, poor lifestyle habits and their consequences, and importance of becoming a role model for patients (14).

With regard to interventions, several researchers tested the effect of interventions on self-care 
practices of students. For example, Stark et al. $(8,15)$ tested the effect of health promotion intervention, while Padykula (17) studied the influence on selfcare practices in response to a holistic nursing course and reflective journaling. Stark et al. (8) tested an intervention consisting of teaching session about the importance of self-care, development of self-care plan, and evaluation of self-care plan over a semester in 82 nursing students, 72 occupational therapy, and 47 speech language pathology students. The speech pathology students were the part of the comparison group and received no intervention. With regard to intervention, significant differences were noted in the health practices concerning overall health promoting lifestyle score $(p=0.014)$, physical activity $(p=0.001)$, and nutrition $(p=0.025)$. Stark et al. (15) encouraged 67 students to develop a lifestyle self-care plan and engage in 2 hours/week self-care practice. The researchers found that this intervention resulted in an improvement of selfcare practices in five domains: Health responsibility $(p=0.001)$, physical activity $(p=0.001)$, nutrition $(p=0.002)$, spiritual growth $(p=0.002)$, and stress management $(p=0.004)$. However, no significant differences were noted in the interpersonal relations domain $(p=0.257)$. Likewise, Padykula (17) found significant pre-post mean differences in the domains: Environment (4.27 vs. 4.35), health responsibility (4.17 vs. 4.32 ), and emotional well-being (4.12 vs. 4.23). Overall, based on these findings, it could be implied that students may find it difficult to engage in self-care due to the above listed factors, but the use of educational and health promotion interventions help in improving their self-care behaviors and practices.

\section{Comparison of self-care practices of nursing and non-nursing students}

The self-care practices of nursing and non-nursing students were compared in three studies $(8,13,14)$. Stark et al. (8) compared 82 nursing students with 72 occupational therapy and 47 speech language pathology students. As previously discussed, speech pathology students were the part of comparison group and received no intervention. The researchers compared the intervention and comparison groups, but no comparison was made between three groups of students. Clément et al. (13) compared practices of nursing students with education students and then made an overall comparison of both nursing students with a baseline study of Quebec population (those comparative findings are beyond the scope of this paper). With regard to nursing students and education students, the researchers noted no significant difference in the health behaviors of nursing students over 3 years $(p \leq 0.05)$ and between nursing and education students $(p \leq 0.05)$. Shriver and Scott-Stiles (14) compared self-care practices of 71 nursing students and 83 non-nursing students in a 2 -year longitudinal study. The number of students decreased in the $2^{\text {nd }}$ year resulting in 57 nursing students and 20 non-nursing students. Therefore, these results should be generalized with caution. Some interesting findings of this study were that non-nursing students (45.8\%) exercised more regularly than nursing students $(22.5 \%)$, but also smoked more than nursing students (non-nursing $=14.5 \%$ and nursing $=7.0 \%$ ). Significant improvements were seen in the self-care behaviors and practices of nursing students compared to non-nursing students in the domains: Eating habits $(p=0.05)$ and self-breast examination $(p=0.009)$. Overall, with one positive and one negative finding, it was difficult to conclude whether nursing students' self-care practices were better than non-nursing students.

\section{Comparison of self-care behaviors and practices across academic years}

Direct comparison of students' practices across different academic years was not made, but several studies compared the self-care practices across semesters and over 2 or 3 years $(1,8,10,11,13,14,17)$. For example, Ashcraft and Gatto (1) reported that no significant difference was noted among the nursing students as they progressed through different years $(p=0.72)$. Stark et al. (8) collected data at two points in time, Semester I (T1) and Semester II (T2), and noted significant differences between TI and T2 scores in the domains: Health responsibility $(p=0.027)$, physical activity $(p=0.017)$, and nutrition $(p=0.047)$. Contrary to these findings, Clément et al. (13) and Nevins and Sherman (9) did not note any statistically significant differences in self-care practices of students across academic years. Padykula (17) also assessed differences in students' understanding of 
self-care practices at 3 times: (i) At the beginning of the holistic nursing course, (ii) at the mid, and (iii) at the end of the course. The researchers reported significant differences at three points in time, but these findings cannot be substituted for self-care practices of students because of the focus on knowledge levels rather than attitudes. Overall, these findings indicated that none of the studies directly compared the differences in self-care practices across years. Hence, no conclusion can be drawn.

\section{DISCUSSION AND AREAS FOR FUTURE RESEARCH}

This literature review explored self-care behaviors and practices of nursing students in general as well as across the academic years of the study and identified areas for the future research. The review of literature indicted that there are limited number of studies conducted to explore self-care practices and behaviors of nursing students. An interesting pattern in the reviewed studies was the inclusion of more female nursing students than male students. This could limit the findings of the studies only to female population. Therefore, the future studies should recruit an equal number of male and female students or should only focus on male nursing students. Furthermore, the future studies should use large, random, and representative samples. The future studies could also employ mixed-method approaches because the use of only quantitative studies and self-administered instruments for data collection could have provided an incomplete understanding of students' self-care practices.

The general conclusions drawn from the reviewed studies are that nursing students understand the importance of self-care for personal well-being and realized the importance of maintaining their diet, sleep, and activity level to improve physical and physiological health. They tend to refrain from tobacco, alcohol, and illegal drug use and pay attention to their personal safety. Nursing students also engage in self-screening practices. However, further research is needed to explore general health screening practices of students because reviewed studies provided limited evidence in this area. These studies focused on exploring screening practices related to Pap smear, self-breast, and self-testicular examination and did not explore general screening practising such as regular dental checkups, stress and depression testing, diabetic testing, blood pressure monitoring, blood work, and so forth. Furthermore, the findings concerning self-testicular examination are not generalizable because of the limited number of male students in the sample.

Although the studies reported factors and interventions that may influence self-care practices of nursing students, further correlational research is needed to explore the strength of relationship of these factors. Further research is also needed to study the effect previously discussed interventions through more robust experimental studies such as randomized control trials (RCT). Future RCTs should include nursing students as control or comparison group rather than non-nursing students which may help in reducing any possible biases due to matching of characteristics of comparison and control groups. Since none of the studies directly compared the selfcare practices and behaviors of students across different academic years, further research is needed to fill this research gap too. Further research is also needed to explore the knowledge, attitudes, and practices of students regarding complementary therapies.

\section{Limitations}

This review is subject to several limitations: (i) Literature search within four databases only and inclusion of limited studies could have resulted in excluding other relevant studies, thereby providing an incomplete understanding of students self-care practices, (ii) the exclusion of dissertations and theses could have also limited an in-depth understanding, and (iii) the thematic analysis of self-care practices and behaviors could have been guided by any pertinent theoretical and conceptual framework.

\section{CONCLUSION}

Nursing students understood the importance of self-care for personal physical, psychological, and emotional well-being and realized the importance of maintaining their diet, sleep, and activity level to improve physical and physiological health. They tend to refrain from tobacco, alcohol, and illegal drug use, pay attention to their personal safety, and focus on several health screening practices including Pap smear, self-breast, and self-testicular examination. 
However, students tend to neglect self-care practices which could improve their emotional and psychological health because of several factors such as academic stress, workload, and inadequate knowledge about the strategies to improve self-care in this domain. These seem to be limited evidence for drawing any conclusions regarding students' use of complementary therapies for self-care, the difference between self-care practices of nursing and non-nursing students, the usefulness of different interventions for improving students' self-care practices, and difference in self-care practices and behaviors of students across academic years. Therefore, future research is needed in these areas.

\section{CONFLICT OF INTERESTS}

Authors declare no conflict of interests.

\section{REFERENCES}

1. Ashcraft PF, Gatto $S L$. Care-of-self in undergraduate nursing students: A pilot study. Nurs Educ Perspect 2015;36(4):255-6.

https://doi.org/10.5480/13-1241.

2. Younas A. A foundational analysis of dorothea orem's self-care theory and evaluation of its significance for nursing practice and research. Creat Nurs 2017;23(1):13-23.

https://doi.org/10.1891/1078-4535.23.1.13.

3. Austen M. Self-care in Nursing: A Call to Action; 2015. Available from: https://www.arnbc.ca/blog/self-care-in-nursing-a-call-to-action-by-marenausten-bsn-student. [Last cited on $2017 \mathrm{Feb} 27$ ].

4. Mills J, Wand T, Fraser JA. On self-compassion and self-care in nursing: selfish or essential for compassionate care? Int J Nurs Stud 2015;52(4):791-3.

https://doi.org/10.1016/j.jnurstu.2014.10.009.
5. Pulido-Martos M, Augusto-Landa JM, Lopez-Zafra E. Sources of stress in nursing students: A systematic review of quantitative studies. Int Nurs Rev 2012;59(1):15-25.

https://doi.org/10.1111/j.1466-7657.2011.00939.x.

6. Younas $A$. Levels of stress and coping strategies used by nursing students in Asian countries: An integrated literature review. J Middle East North Afr Sci 2016;2(4):50-7.

https://doi.org/10.12816/0032673.

7. Clark CS. Stress, psychoneuroimmunology and self-care: What every nurse needs to know. J Nurs Care 2014;3(2):146.

https://doi.org/10.4172/2167-1168.1000146.

8. Stark MA, Hoekstra T, Hazel DL, Barton B. Caring for self and others: Increasing health care students' healthy behaviors. Work 2012;42(3):393-401.

9. Nevins CM, Sherman J. Self-care practices of baccalaureate nursing students. J Holist Nurs 2016;34(2):185-92.

https://doi.org/10.1177/0898010115596432.

10. Haddad L, Kane D, Rajacich D, Cameron S, Al-Ma'aitah R. A comparison of health practices of Canadian and Jordanian nursing students. Public Health Nurs 2004;21(1):85-90.

https://doi.org/10.1111/j.1525-1446.2004.21112.x.

11. Horneffer KJ. Students' self-concepts: implications for promoting self-care within the nursing curriculum. J Nurs Educ 2006;45(8):311-6.

12. Chow J, Kalischuk RG. Self-care for caring practice: Student nurses' perspectives. Int J Hum Caring 2008;12(3):31-7.

13. Clément M, Jankowski LW, Bouchard L, Perreault M, Lepage Y. Health behaviors of nursing students: a longitudinal study. I Nurs Educ 2002;41(6):257-65.

14. Shriver $C B$, Scott-Stiles $A$. Health habits of nursing versus non-nursing students: a longitudinal study. J Nurs Educ 2000;39(7):308-14.

15. Stark MA, Manning-Walsh J, Vliem S. Caring for self while learning to care for others: a challenge for nursing students. J Nurs Educ 2005;44(6):266-70.

16. Siappo CL, Núñez YR, Cabral IE. Nursing students experiences in self-care during training process in a Private University in Chimbote, Peru. Escola Anna Nery 2016;20(1):17-24.

https://doi.org/10.5935/1414-8145.20160003.

17. Padykula BM. RN-BS students' reports of their self-care and health-promotion practices in a holistic nursing course. J Holist Nurs 2016. pii:0898010116657226. 